\title{
Observation of Metastable and Stable Energy Levels of EL2 in Semi-insulating GaAs
}

\author{
D. Kabiraj ${ }^{\star}$ and Subhasis Ghosh ${ }^{\dagger}$ \\ * Nuclear Science Centre, New Delhi 110067 and \\ ${ }^{\dagger}$ School of Physical Sciences, Jawaharlal Nehru University, New Delhi 110067
}

\begin{abstract}
By using combination of detailed experimental studies, we identify the metastable and stable energy levels of EL2 in semi-insulating GaAs. These results are discussed in the light of the recently proposed models for stable and metastable configurations of EL2 in GaAs.

PACS numbers: 71.55.Eq, 72.80.Ey, 78.55.Cr
\end{abstract}

Physics of metastable point defects is one of the most fascinating problems in contemporary condensed matter physics. Over the years several metastable defects in both covalent and ionic solids have been discovered, but for technological reasons, EL2 in GaAs is the most intensely studied, yet least understood, metastable defect. It is believed [1] that bond-breaking mechanism(BBM), in which defects change lattice position is universally responsible for all the metastable defects in solids. The EL2 defects [2], responsible for semi-insulating(SI) properties of GaAs by compensating residual acceptors, can exists in two different atomic configurations. The first one is the well characterized normal configuration $\left(\mathrm{EL}^{n}\right)$ and the second one is the metastable configuration $\left(\mathrm{EL}^{m}\right)^{m}$ in which all the optical, electrical and magnetic properties of EL2 disappear when SI-GaAs is illuminated with a subband gap light $\sim 1.1 \mathrm{eV}$ at low temperature $(\leq 150 \mathrm{~K})$, known as phtoquenching(PQ). EL2 ${ }^{m}$ is metastable because all the properties can be recovered either by heating the sample, or by phtoexcitation at low temperature, known as photorecovery(PR). Though, a great deal of attention has already been paid to understand the physics of this defect, but the microscopic origin of EL2 remains illusive till today. In particular, the most important issues remained to be resolved are, (i) atomic configuration of EL2 in both normal and metastable configurations, (ii) driving mechanism for EL2 ${ }^{n}$ to EL2 ${ }^{m}$ transition and, (iii) compensation mechanism after PQ, since free holes with concentration same as that of EL2 will be available when EL2s are in photoquenched state. There are some efforts [3, 4, [5, 6] to explain some of these issues by postulating the existence of actuator levels which trigger the metastable transition by capturing photoexcited holes from EL2s. But, this actuator level is characterized by the absence of any direct experimental observation. In addition to this, interest in EL2 has been revived for two reasons, first: an increasing interest in defect engineering using this defect and second: the role of this defect on the properties of low temperature grown GaAs.

Several models [2] based on either isolated native point defect or defect complex comprising of arsenic anitisite $\left(\mathrm{As}_{G a}\right)$, gallium antisite $\left(\mathrm{Ga}_{A s}\right)$, arsenic vacancy $\left(\mathrm{V}_{A a}\right)$, gallium $\operatorname{vacancy}\left(\mathrm{V}_{G a}\right)$, arsenic interstitial $\left(\mathrm{As}_{i}\right)$ have been proposed, but very little is known about the electrical properties and defect energy levels of EL2 ${ }^{m}$. Recently, two models, for the first time, proposed correlation between electrical activity and the defect energy level related to metastable configuration of EL2. Fukuyama et al [7] have proposed a BBM-based model and shown that a three-center-complex $\mathrm{V}_{A s^{-}} \mathrm{As}_{\mathrm{Ga}^{-}}$ $\mathrm{Ga}_{A s}$ is the atomic origin of EL2 with specific predictions of different defect energy levels related to EL2 in normal and metastable configurations and their role on $\mathrm{PQ}\left(\mathrm{EL} 2^{n}\right.$ $\left.\rightarrow \mathrm{EL}^{m}\right)$ and $\mathrm{PR}\left(\mathrm{EL} 2^{m} \rightarrow \mathrm{EL}^{n}\right)$. Chadi [8] has also proposed a BBM-based model and reaffirmed that atomistic origin of EL2 is the isolated $\mathrm{As}_{G a}$ which can exist in eight charge states giving rise to two energy levels for $\mathrm{EL} 2^{n}$ and EL2 ${ }^{m}$. It is only known that EL2 introduces a mid-gap level. There is no experimental investigations on the normal and metastable levels proposed in Ref. [7] and Ref. 8]. Identification of these levels will help immensely to resolve a long standing controversy regarding the microscopic origin of EL2 ${ }^{n}$ and EL2 ${ }^{m}$ and mechanism behind the EL2 ${ }^{n} \rightarrow \mathrm{EL}^{m}$ transition. In this letter, we report an experimental observation of the energy levels directly related to EL2 in normal and metastable states using different spectroscopic techniques suitable for highly resistive materials like, SI-GaAs.

Experiment. The photo-current (PC), thermally stimulated current (TSC), photo-Hall-voltage (PHV) and thermally stimulated Hall voltage (TSHV) measurements on both unirradiated and irradiated SI-GaAs samples obtained from two different sources were carried out. The observation of TSC and TSHV spectra with the signature of trap levels requires enough carriers be available for capture and finally for emission and is achieved by the photoexcitation at low temperature prior to heating cycle. Three monochromatic sources, $1.16 \mathrm{eV}$ and $1.37 \mathrm{eV}$ (which cause PQ) and $2.54 \mathrm{eV}$ (which does not cause PQ) 4, 9] were used for this purpose. In order to perform the TSC and TSHV measurements under identical initial condition, following sequence was used, (i) the sample was cooled to $10 \mathrm{~K}$ in dark and equilibrated for two hours and then photoexcited for $\mathrm{PC}$ growth and finally illumination was terminated at different times for obtaining the TSC spectra from $10 \mathrm{~K}$ to $300 \mathrm{~K}$ by heating the sample at the rate of $0.1 \mathrm{~K} / \mathrm{sec}$, (ii) the sample temperature was raised to $320 \mathrm{~K}$ and equilibrated for two hours to avoid any resid- 
ual metastability, (iii) the sample temperature was again brought down to $10 \mathrm{~K}$ and step (i) was followed. To support the microscopic identification, $48 \mathrm{MeV} \mathrm{Li} \mathrm{ions,} \mathrm{ob-}$ tained from the Pelletron accelerator at Nuclear Science Centre, New Delhi, was used to create and annihilate the intrinsic point defects in SI-GaAs. The energy is chosen such a way that the range of the ions is larger than the thickness $(\sim 100 \mu m)$ of the sample, so that the damage of the sample due to the creation of extended defects by nuclear collision can be avoided and the intrinsic defects are created only by electronic excitation 10 .

Fig.1(a) shows the PC growth at $10 \mathrm{~K}$ under the excitation of $1.16 \mathrm{eV}$ and $2.54 \mathrm{eV}$ lights. As expected there is no PQ of EL2 in case of $2.54 \mathrm{eV}$ light, but strong $\mathrm{PQ}$ has been observed after 50sec of photoexcitation in case of $1.16 \mathrm{eV}$ light and subsequently $\mathrm{PC}$ is saturated to a steady state value, which depends on the intensity of the photoexcitation. Fig.1(b) shows the comparison of TSC spectra taken after 20sec exposure with $1.16 \mathrm{eV}$ and $2.54 \mathrm{eV}$ lights, respectively. The most interesting feature is the completely different TSC spectra in these two cases. The peaks at $26 \mathrm{~K}$ and $140 \mathrm{~K}$ are absent in TSC spectra taken with $2.54 \mathrm{eV}$ light and peak at $75 \mathrm{~K}, 240 \mathrm{~K}$ and $260 \mathrm{~K}$ are absent in the TSC spectra taken with $1.16 \mathrm{eV}$ light. Fig.1(c) shows the comparison of TSC spectra taken after 600 sec exposure with $1.16 \mathrm{eV}$ and $2.54 \mathrm{eV}$ lights. In contrast to previous case, TSC spectra are almost identical in this case. Hence, there is no difference in TSC spectra taken with $2.54 \mathrm{eV}$ light for different exposure times, but TSC spectra taken with $1.16 \mathrm{eV}$ light are completely different for different exposure times.

To study the PQ and change in conduction type during quenching, time evolution of $\mathrm{PC}$ and $\mathrm{PHV}$ growth has been studied under the photoexcitation with two different quenching lights $(1.16 \mathrm{eV}$ and $1.37 \mathrm{eV})$, which are shown in Fig.2 and Fig.3. It is known [7] that SI-GaAs is very weakly n-type. The temporal evolution of $\mathrm{PC}$ and PHV can be divided into two regimes, regime I: a steep rise followed by decrease towards a minimum due to $\mathrm{PQ}$ of EL2 $\left(\mathrm{EL} 2^{n} \rightarrow \mathrm{EL} 2^{m}\right)$ and conduction is p-type in this regime, regime II: $\mathrm{PC}$ and $\mathrm{PHV}$ reach a minimum, conduction type changes from p-type to n-type, followed by a very slow increase due to PR of EL2(EL2 $\left.{ }^{m} \rightarrow \mathrm{EL}^{n}{ }^{n}\right)$ and finally conduction type returns to weakly n-type. In case of $1.16 \mathrm{eV}$ light(Fig.2), $\mathrm{PQ}$ is over after 50sec, followed by $\mathrm{PR}$ which restores initial PC and conduction type after $550 \mathrm{sec}$, but in case of $1.37 \mathrm{eV}$ light(Fig.3), it takes longer time $(\sim 1500 \mathrm{sec})$ for $\mathrm{PQ}$ and efficiency of $\mathrm{PR}$ is very low compared to $1.16 \mathrm{eV}$ light. This is due to strong dependence quantum efficiency of PQ and PR on the wavelength of photoexcitation [4, 9]. To investigate the evolution of TSC spectra at different stages of quenching and recovery of EL2, light exposure was terminated at different stages of $\mathrm{PQ}$ and $\mathrm{PR}$ and the corresponding TSC spectra show how the different energy levels evolve with quenching and recovery of EL2. Fig.2 and Fig.3 show similar TSC spectra irrespective of wavelength of initial photoexciation. It is clear from Fig.2 and Fig. 3 that energy levels at $26 \mathrm{~K}$ and $140 \mathrm{~K}$ are disappearing as EL2 $2^{n} \rightarrow \mathrm{EL} 2^{m}$, so these energy levels must be related to metastable configuration of EL2. After PR, these metastable levels are not recovered, instead, a new set of levels at $75 \mathrm{~K}, 240 \mathrm{~K}$ and $260 \mathrm{~K}$ are appeared, so these energy levels are observed as EL2 ${ }^{m} \rightarrow \mathrm{EL} 2^{n}$. Similar metastable TSC peak at around 140K has been previously observed [11, 12], but not the other metastable TSC peaks. As shown in Ref.[9], we have also observed two step PQ(shown in Fig2. and Fig.3) which will be subject of our future investigation.

Fig.4 shows the TSC and TSHV spectra taken after 100sec exposure(during PQ) with of $1.16 \mathrm{eV}$ light. By comparing the TSHV and TSC spectra, we observe, (i) the metastable TSC peaks at $26 \mathrm{~K}$ and $140 \mathrm{~K}$ are present in TSHV spectra and hence these are hole traps [13], (ii) the TSC peaks at $65 \mathrm{~K}, 90 \mathrm{~K}$ and $120 \mathrm{~K}$ are absent, instead a dip at their positions and these should be due to electron traps 13], which should give rise to negative peak in TSHV spectra.

The irradiation induced modifications in PC and TSC measurement results are shown in Fig.5, which provide useful information regarding the role of the levels at $26 \mathrm{~K}$ and $140 \mathrm{~K}$ observed during EL2 ${ }^{n} \rightarrow$ EL2 ${ }^{m}$ transformation. Fig.5(a) shows how efficiency of PQ is reduced compared to that in control sample in samples irradiated with a fluence of $1 \times 10^{12}$ ions $/ \mathrm{cm}^{2}$ and $1 \times 10^{13}$ ions $/ \mathrm{cm}^{2}$. Corresponding TSC spectra in Fig.5(b), clearly show that the heights of the metastable peaks at $26 \mathrm{~K}$ and $140 \mathrm{~K}$ responsible for $\mathrm{PQ}$ and EL2 ${ }^{n} \rightarrow \mathrm{EL} 2^{m}$ transition reduced in sample irradiated with a fluence of $1 \times 10^{12}$ ions $/ \mathrm{cm}^{2}$ and finally TSC peak at $26 \mathrm{~K}$ disappeared and the peak height of TSC peaks at $140 \mathrm{~K}$ reduced further in samples irradiated with a fluence of $1 \times 10^{13}$ ions $/ \mathrm{cm}^{2}$. Hence, there is correlation between the reduction of efficiency of PQ i.e. EL2 concentration and reduction of the peak heights of the metastable peaks at $26 \mathrm{~K}$ and $140 \mathrm{~K}$ related to EL2 ${ }^{m}$.

Origin of TSC/TSHV peaks in SI-GaAs when EL2S are in normal state. The TSC spectra obtained either after photoexcitation of $2.54 \mathrm{eV}$ light or after photoexcitation of $1.16 \mathrm{eV}$ (or $1.37 \mathrm{eV}$ )for long duration(after PR) are the energy levels in SI-GaAs when EL2s are in normal state. The origin of weak TSC peak at 40K is not known much, recently it has been shown [14] that it may be due to complex related to $\mathrm{V}_{G a}$ and $\mathrm{Ga}_{A s}$. The strong peak at $65 \mathrm{~K}$ and $120 \mathrm{~K}$ (as shown in Fig.1, Fig.2 and Fig.3) are due to two energy levels of double donor $\mathrm{V}_{A s}(+/ 0$ and $2+/+)[11,12]$. We have shown [16] that the strong peak at $90 \mathrm{~K}$ may be due to $\mathrm{O}_{A s}$. This identification is corroborated by the annihilation of this peak under ion irradiation with $50 \mathrm{MeV} \mathrm{Li}$, as shown in Fig.5. Based on earlier published data 11, 12, 15] and energetic positions, the TSC peaks at $150 \mathrm{~K}, 200 \mathrm{~K}$ and $215 \mathrm{~K}$ are attributed 
to $\mathrm{As}_{i}-\mathrm{V}_{A s}, \mathrm{Ga}_{A s}-\mathrm{V}_{A s}$ and $\mathrm{As}_{G a^{-}}-\mathrm{V}_{A s}$ complexes, respectively. This is all about the origin of TSC peaks when EL2s are in normal state, except the peaks at $240 \mathrm{~K}$ and $260 \mathrm{~K}$, which will be discussed later.

Origin of metastable levels of EL2. All these findings indicate that the metastable TSC peaks at $26 \mathrm{~K}(\sim 0.035 \mathrm{eV}), 132 \mathrm{~K}(\sim 0.27 \mathrm{eV})$, which are observed during EL2 ${ }^{n} \rightarrow \mathrm{EL}^{m}$ are related to EL2 ${ }^{m}$, i.e. EL2 in metastable configuration, because of the (i) observation of these levels when EL2s are in metastable state, (ii) disappearance of these levels when EL2s are in normal state, (iii) gradual disappearance of these levels when the concentration of EL2 are reduced in irradiated samples, (iv) similar electrical property of all these levels, which are hole-traps, and (v) absence of these levels in Cr-doped SI-GaAs(not shown). These results can be interpreted by both the models discussed in Ref. [7] and Ref. [8]. But, to put our experimental results on more rigorous basis we now discuss the three-center-complex model based on the proposal of $\mathrm{V}_{A s}-\mathrm{As}_{G a}-\mathrm{Ga}_{A s}$, as atomistic origin of EL2. According to this model, (i) $A s_{G a}-V_{A s}$ pair is responsible for metastability while $G a_{A s}$ controls the transition between the normal and metastable state, (ii) the Columbic interaction between $G a_{A s}^{-}$and $A s_{G a}^{+}$ pins the As atom at its Ga-site. PQ starts with the photoionization of hole from $A s_{G a}^{+}\left(A s_{G a}^{+} \rightarrow A s_{G a}^{0}+h\right)$ and subsequent capture of hole by $G a_{A}^{-}$, which is supported by the p-type conductivity during $\mathrm{PQ}$, as shown in Fig.2 and Fig.3. This results the neutralization of $G a_{A s}^{-}$ and $A s_{G a}^{+}$to $G a_{A s}^{0}$ and $A s_{G a}^{0}$, respectively, leading to (i) switching off the Coulombic attraction and breakage of the bond between $A s_{G a}$ and $G a_{A s}$ and (ii) As atom moves towards the $\mathrm{V}_{A s}$ due to $\mathrm{BBM}$ until the formation of metastable complex. Movement of As atom from $\mathrm{As}_{\mathrm{Ga}}$ site towards the interstitial site gives rise to $\mathrm{V}_{G a}$ and $\mathrm{As}_{i}$. The role of $\mathrm{As}_{G a}$ and movement of $\mathrm{As}_{i}$ by few angstroms on the EL2 $2^{n} \rightarrow \mathrm{EL}^{m}{ }^{m}$ transition has also been established theoretically $\left[8\right.$, 17]. Hence, as EL2 ${ }^{n} \rightarrow \mathrm{EL}^{m}{ }^{m}$, these metastable point defects $\left(\mathrm{V}_{G a}\right.$ and $\left.\mathrm{As}_{i}\right)$ should give rise to TSC peaks, which should be hole traps. The metastable defect energy levels at $0.035 \mathrm{eV}$ and $0.27 \mathrm{eV}$ from valence band minimum(VBM) are attributed to $\mathrm{As}_{i}$ and $V_{G a}$, respectively. Similar ionization energies of $\mathrm{V}_{G a}$ and $\mathrm{As}_{i}$ have been predicted by calculating local atomic structure with lattice relaxation $[18,19]$. The existence of $\mathrm{V}_{G a}$ in the metastable configuration of EL2 $2^{m}$ has been argued by positron annihilation experiment [20].

The recovery of EL2 according to this three-centercomplex model would be $\left[V_{A s}-(A s)-V_{G a}\right]-G a_{A s}^{0} \rightarrow$ $\left[V_{A s}^{+}-A s_{G a}^{+}\right]-G a_{A s}^{-}+e$, and the excess electrons in the conduction band during this process is consistent with experimentally observed(Fig.2 and Fig.3) conduction type conversion from p-type during $\mathrm{PQ}$ to n-type during recovery of EL2 7]. According to this model, three processes: (1) ionization of deep donor $V_{A s}^{0}$, which is supported by the enhancement of TSC peaks at $65 \mathrm{~K}$ and $120 \mathrm{~K}$ dur- ing PR, as shown in Fig.2 and 3, (2) ionization of deep acceptor $G a_{A s}^{0}$, which should result a new metastable peak during PR and is attributed to the TSC peak at $75 \mathrm{~K}(\sim 0.13 \mathrm{eV})$, similar ionization energy of $\mathrm{Ga}_{A s}(0 /-)$ has also been predicted theoretically [21, 22], and (3) movement of As atom towards the antisite position resulting annihilation of $\mathrm{As}_{i}$ and $\mathrm{V}_{G a}$, which is supported by the disappearance of TSC peaks at $26 \mathrm{~K}$ the $140 \mathrm{~K}$ during PR.

Origin of stable levels of EL2. As the recovery process proceeds with prolonged photoexcitation with sub band gap light, we observe the disappearance of metastable levels related to EL2 and appearance of new peaks at $240 \mathrm{~K}(\sim 0.49 \mathrm{eV})$ and $260 \mathrm{~K}(\sim 0.56 \mathrm{eV})$. These peaks are always observed when TSC is taken with above band gap light. These levels are related to the normal state of EL2. Based on their energetic positions, these levels are attributed to double acceptor $\mathrm{Ga}_{A s}(-/ 2-)$ and double donor $\operatorname{As}_{G a}(2+/+)[18,19]$. To have EL2s in normal state, Coulombic interaction is required between $\mathrm{Ga}_{A s}$ and $\mathrm{As}_{G a}$, so they should be either in $\mathrm{Ga}_{A s}^{-}$and $\mathrm{As}_{A s}^{+}$ or $\mathrm{Ga}_{A s}^{--}$and $\mathrm{As}_{A s}^{++}$states. Recently, similar ionization energy, $0.47 \mathrm{eV}$ from VBM, which should be equivalent to TSC peak at $240 \mathrm{~K}$, has been observed by excitation photocapacitance method 23]. Lastly, we would like raise a issue regarding the observation of these stable levels only when EL2s are always in normal state i.e. never photoquenched at low temperature. Once the temperature is raised beyond $150 \mathrm{~K}$, they should be observed always. Is the thermal recovery not complete beyond $150 \mathrm{~K}$ ? Self promoted behavior of EL2 and temperature dependent incubation time have been observed [7] during recovery and explained by some kind of correlation among EL2s, but more experimental and theoretical studies are required for the ascertainment of this speculation. It has been shown that correlated defects are required for consistent description of experimental results in highly compensated Ge 24 and magnetic semiconductor (Ga,Mn)As 25.

In conclusion, we have identified the defect energy levels related to normal and metastable configurations of EL2 in SI-GaAs by using different spectroscopic techniques and $\mathrm{PC}$ growth during $\mathrm{PQ}$ and $\mathrm{PR}$ under different initial conditions. A three center model for microscopic structure of EL2 has been discussed in the context of the origin of these metastable and stable levels and their role on PQ and PR of EL2 in SI-GaAs.

[1] J. Nissila et al, Phys. Rev. Lett. 82, 3276 (1999).

[2] D. Redfield and R. H. Bube, Photoinduced Defects in Semiconductors(Cambridge University Press, New York, 1996).

[3] T. Benchiguer et al, Jpn. J. Appl. Phys. Part 1, 31, 2669(1992). 
[4] A. Alvarez, J. Jimenez and M. A. Gonzalez, Appl. Phys. Lett. 682959 (1996).

[5] T. Ohyama et al, Jpn. J. Appl. Phys. Part 1, 32 1889(1993).

[6] M. Suemitsu, H. Takahashi and N. Miyamoto, Phys. Rev. B 52 1666(1995).

[7] A. Fukuyama, T. Ikari, Y. Akashi and M. Suemitsu, Phys. Rev. B 67 113202(2003).

[8] D. J. Chadi, Phys. Rev. B, 68, 192304(2003).

[9] V. Pandian and V. Kumar, J. Appl. Phys. 70, 5114 (1991).

[10] J. F. Ziegler, J. P. Biersac and U. Littmark, The Stopping and Range of Ions in Matter(Pergamon, New York, 1985).

[11] Z. Q. Fang and D. C. look, J. Appl. Phys. 73, 4971(1993).

[12] D. C. Look et al, Phys. Rev. B 55, 2214(1997).

[13] R. Kiliulis, V. Kazukauskas and J. C. Bourgoin, J. Appl. Phys. 79, (6951) 1996.

[14] H. G. Svavarsson, J. T. Gudmundsson and H. P. Gislason, Phys. Rev. B 69, 155209 (2004).

[15] M. Pavlovic, U. V. Desnica, J. Gladic, J. Appl. Phys. 88 4563 (2000).

[16] D. Kabiraj and S. Ghosh, Appl. Phys. Lett. 84, 1713 (2004).

[17] D. J. Chadi and K. J. Chang, Phys. Rev. Lett. 60, 2187(1988).

[18] H. Seong and L. J. Lewis, Phys. Rev., B 52, 5675(1995).

[19] J. T. Schick, C. G. Morgan and P. Papoulias, Phys. Rev. B 66, 195302 (2002).

[20] R. Krause etal, Phys. Rev. Lett. 65, 3329 (1990).

[21] M. J. Puska, J. Phys. Condens Matter, 1, 7347 (1989).

[22] S. B. Zhang and D. J. Chadi, Phys. Rev. Lett. 64, 1789 (1990).

[23] Y. Oyama and J. Nishizawa, J. Appl. Phys. 97, 033705 (2005).

[24] K. M. Itoh et al, Phys. Rev. B, 53, 7797 (1996).

[25] C. Timm, F. Scafer and F. von Oppen, Phys. Rev. Lett. 89, 137201 (2002).

\section{Figure Captions}

Figure 1. (a) PC growth in SI-GaAs with $1.16 \mathrm{eV}$ light(solid line) and $2.54 \mathrm{eV}$ light(dashed line). Photoex- citation terminated at different times(shown by $\Uparrow$ in (a)) and corresponding TSC spectra taken with $1.16 \mathrm{eV}$ and $2.54 \mathrm{eV}$ lights for (b) 20sec and (c) 500sec exposure at $10 \mathrm{~K}$. Light was switched on at $\mathrm{t}=10 \mathrm{sec}$.

Figure 2. (a) Comparison of temporal evolution of PC growth and photo Hall voltage(PHV) under the photoexcitation of $1.16 \mathrm{eV}$ light. Light was switched on at $\mathrm{t}=10$ sec. (b) Evolution of TSC spectra under the different dose of initial photoexcitation(shown by $\Uparrow$ in (a)). TSC peaks observed during $\mathrm{PQ}\left(\mathrm{EL} 2^{n} \rightarrow \mathrm{EL} 2^{m}\right)$ and $\mathrm{PR}\left(\mathrm{EL} 2^{m} \rightarrow \mathrm{EL} 2^{n}\right)$ are indicated by $\uparrow$ and $\downarrow$, respectively. TSC spectra(except the bottom one) are shifted up for clarity.

Figure 3. (a) Comparison of temporal evolution of PC growth and photo Hall voltage(PHV) under the photoexcitation of $1.37 \mathrm{eV}$ light. Light was switched on at $\mathrm{t}=10$ sec. (b) Evolution of TSC spectra under the different dose of initial photoexcitation(shown by $\Uparrow$ in $(\mathrm{a})$ ). TSC peaks observed during $\mathrm{PQ}\left(\mathrm{EL} 2^{n} \rightarrow \mathrm{EL}^{m}\right)$ and $\mathrm{PR}\left(\mathrm{EL} 2^{m} \rightarrow \mathrm{EL} 2^{n}\right)$ are indicated by $\uparrow$ and $\downarrow$, respectively. TSC spectra(except the bottom one) are shifted up for clarity.

Figure 4. Comparison of TSC(solid line) and TSHV(dotted line) spectra during PQ i.e. when EL2s are in metastable states. The peaks related to EL2 ${ }^{m}$ are indicated by $\operatorname{arrow}(\uparrow)$.

Figure 5. (a) PC growth in irradiated SI-GaAs samples with $1.37 \mathrm{eV}$ light. Light was switched on at $\mathrm{t}=10 \mathrm{sec}$. (b) Evolution of TSC spectra in irradiated SI-GaAs samples, with 20 sec initial photoexposure. 

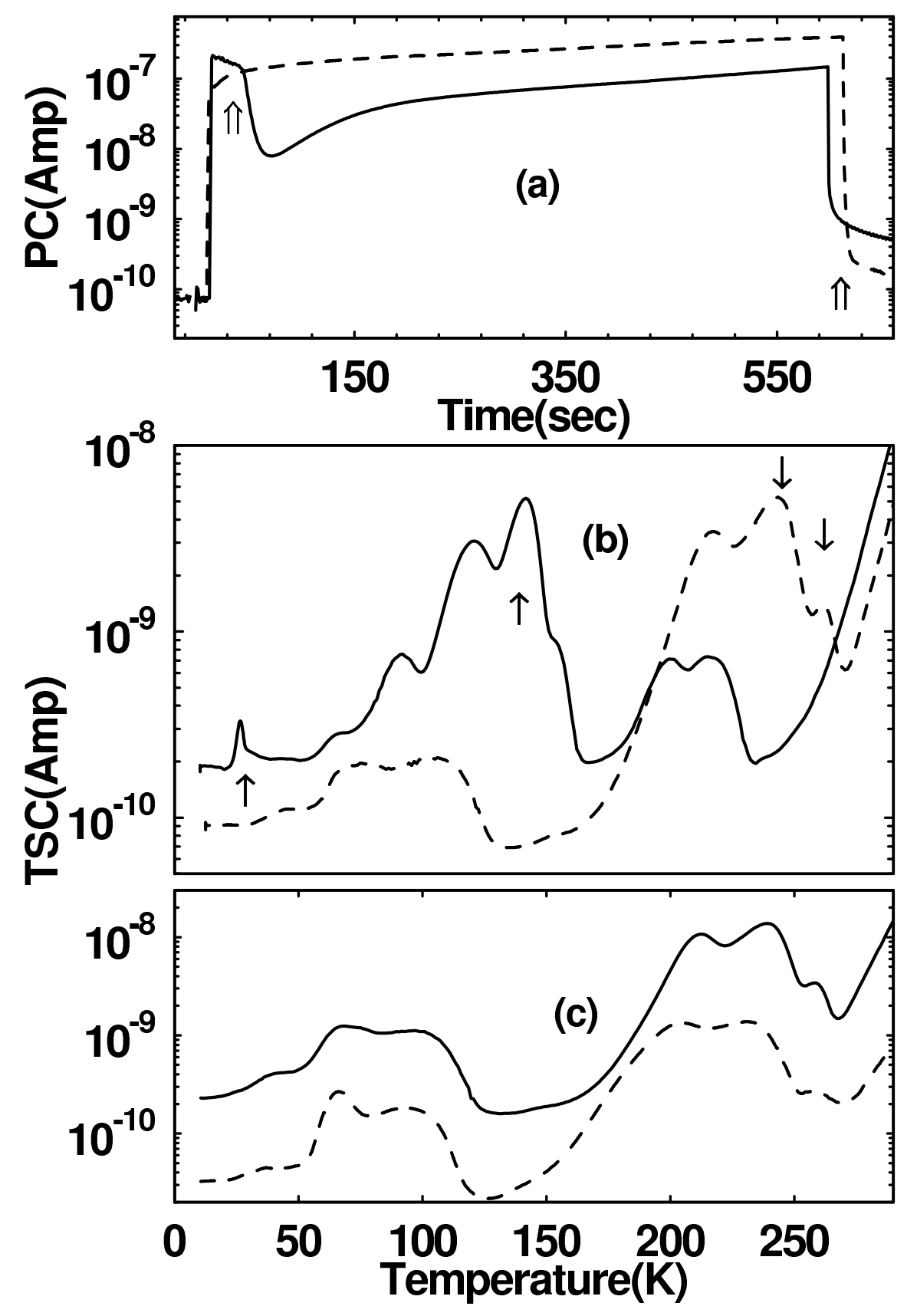

Figure 1 

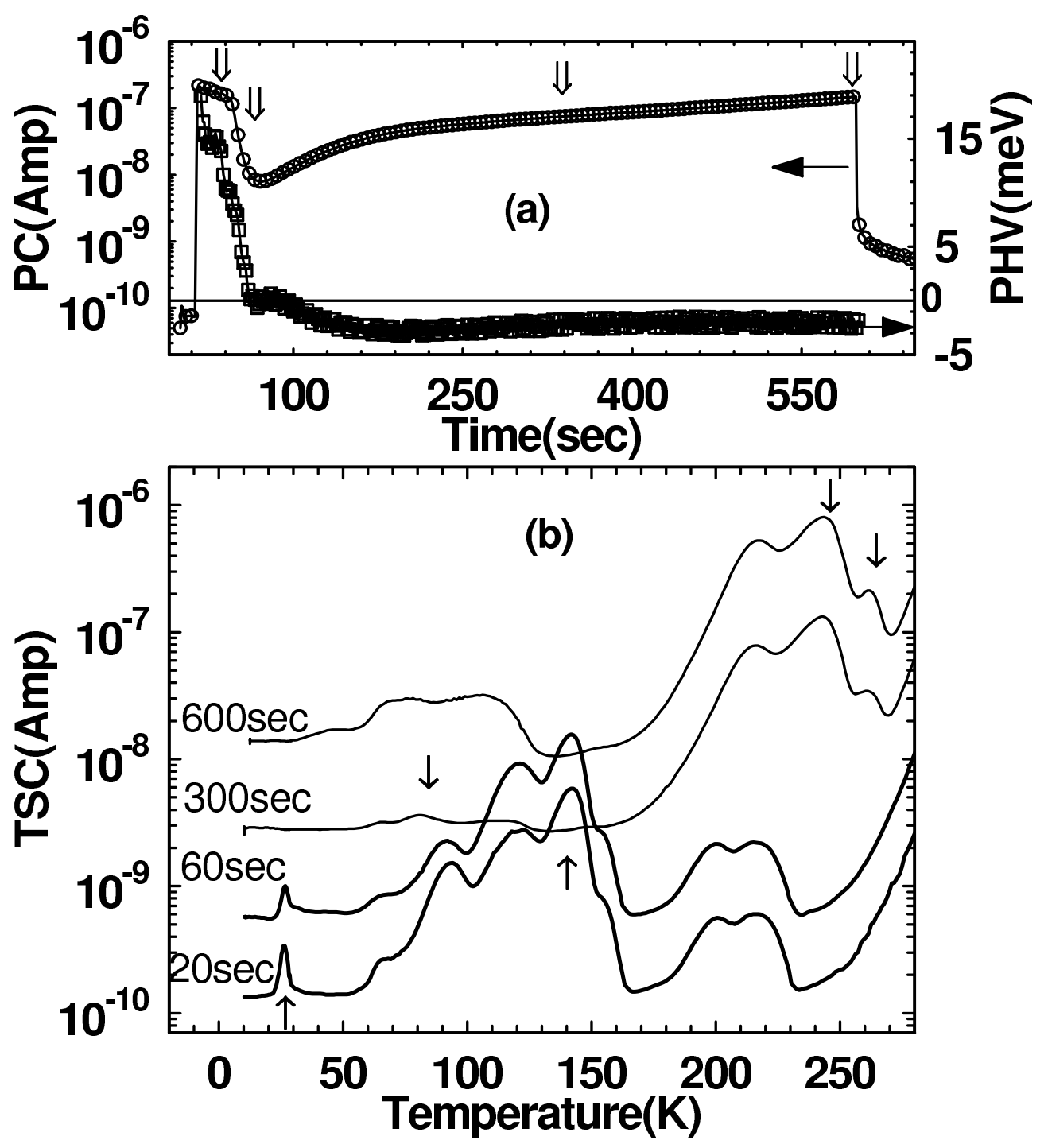

Figure 2 

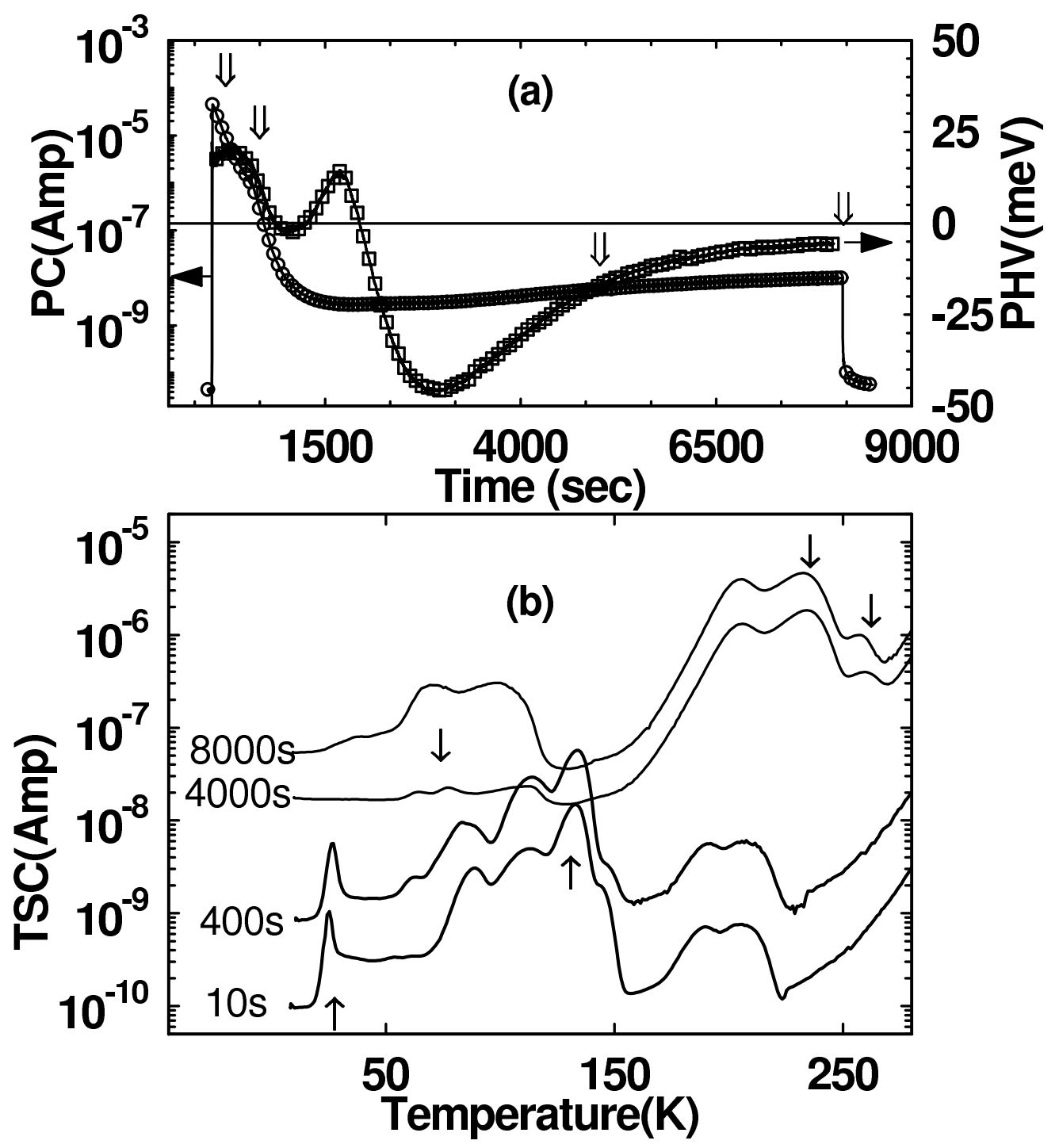

Figure 3 


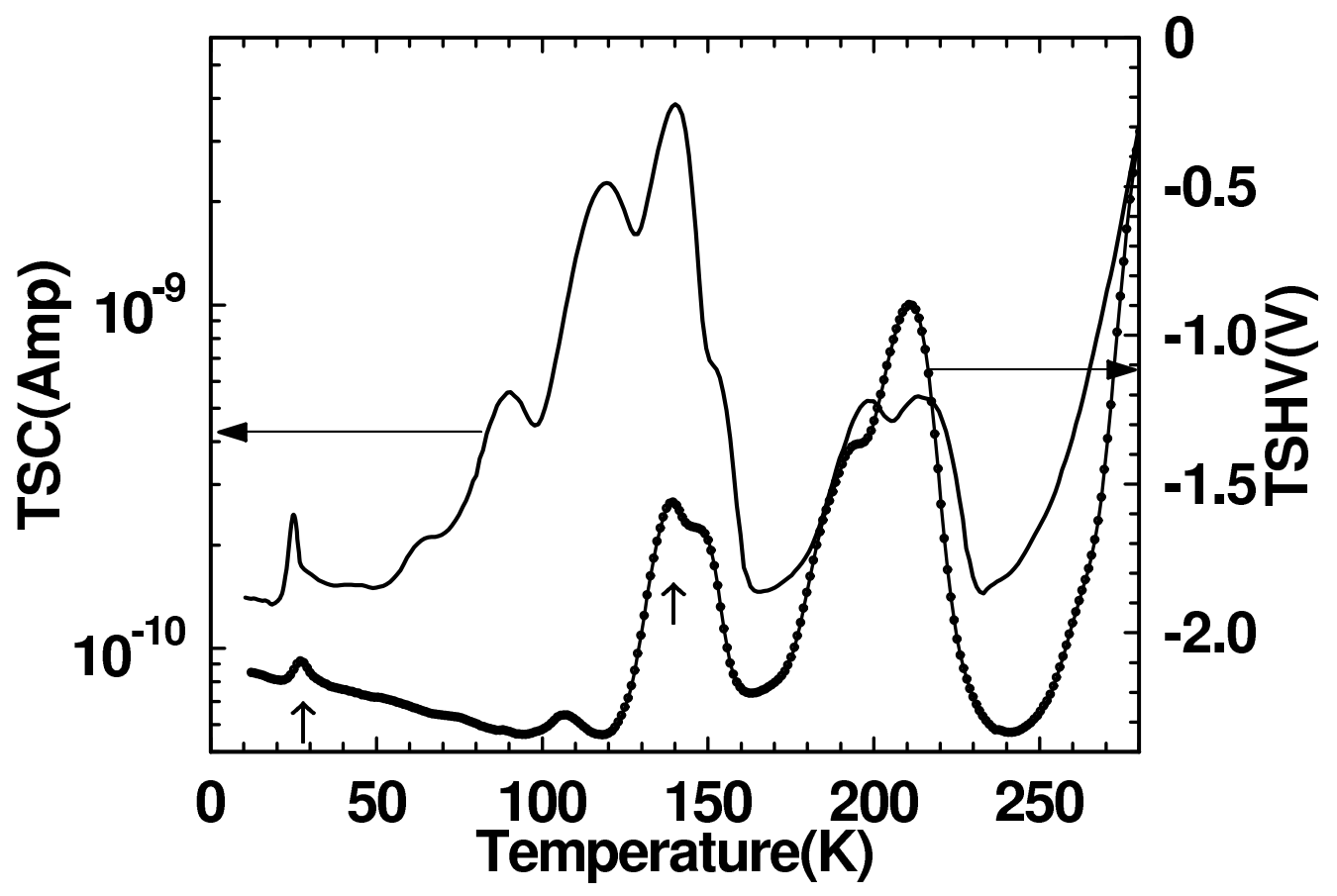

Figure 4 

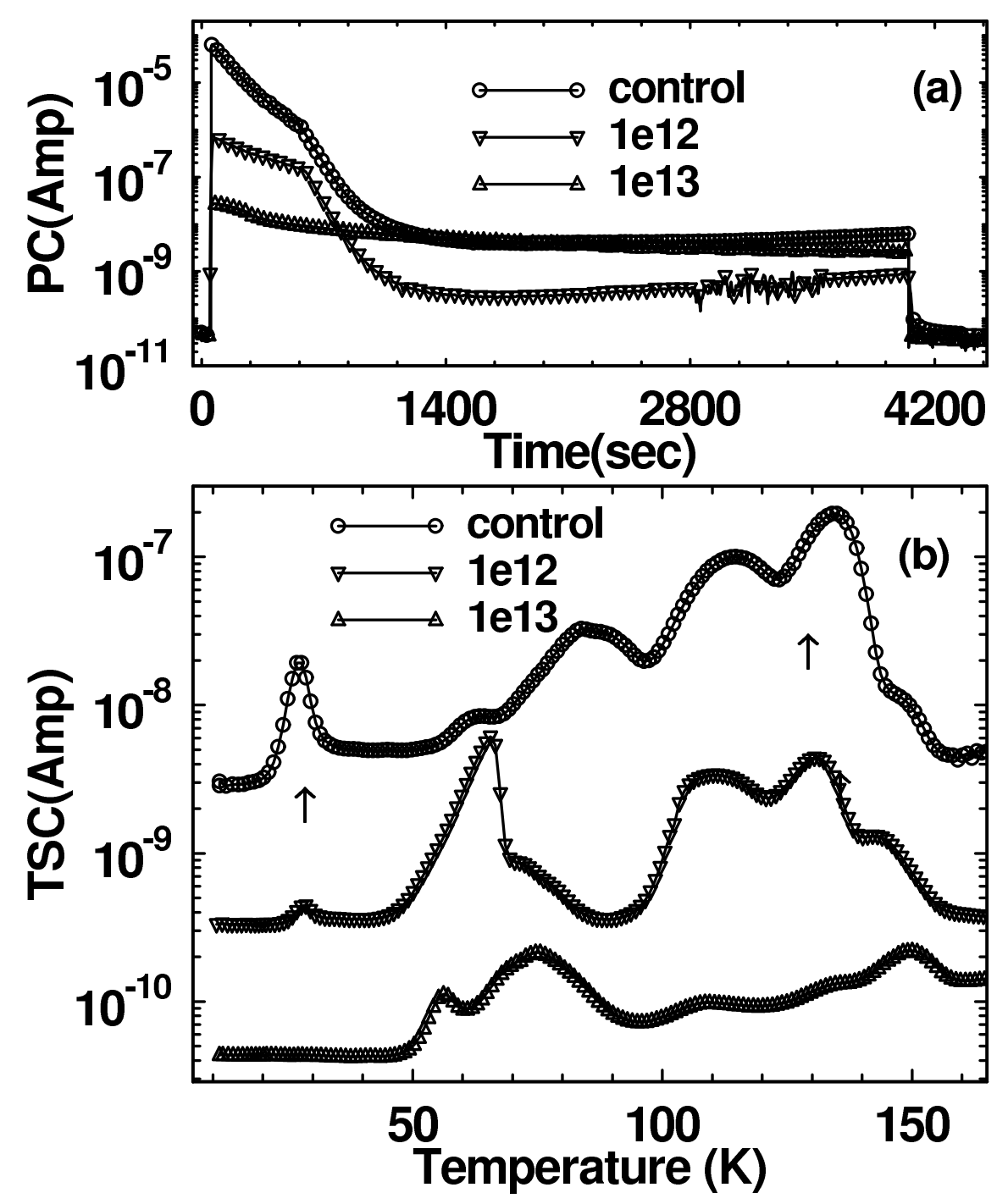

Figure 5 